\title{
AVALIAÇÃO SENSORIAL DE PASTÉIS FRITOS EM ÓLEO DE SOJA COM DIFERENTES TEMPOS DE AQUECIMENTO
}

PEDRO RAMOS DA COSTA NETO *

RENATO JOÃO SOSSELA DE FREITAS

NINA WASZCZYNSKYJ **

\begin{abstract}
Verificou-se a influência do aquecimento do óleo de soja na aceitabilidade de produto frito. Pastéis de queijo fritos em óleo aquecido a fogo direto por 1,$5 ; 18 ; 30$ e 36 horas foram avaliados por dezoito julgadores através do método de escala hedônica de nove pontos. Os resultados mostraram que o óleo de fritura não deve ser reutilizado por mais de 30 horas de aquecimento constante.
\end{abstract}

Os óleos e gorduras submetidos ao aquecimento contínuo, no processo de frituras por imersão em lanchonetes restaurantes, sofrem alterações que a curto prazo modificam sua tonalidade, aumentam sua viscosidade e acidez, diminuindo o Indice de iodo $(1,2,7,9,10,13,17,18,20,25,27$, $29,30)$. Ocorre a formação de substâncias voláteis que dão origem a aromas e sabores desagradáveis $(14,15,21,23)$, popularmente conhecidos por ranço (2) e contribuem com efeitos tóxicos, quando da ingestão contínua e prolongada de produtos rancificados $(3,5,6,8,11,20)$.

o óleo usado nas frituras constitui importante ingrediente do produto processado, influindo em suas características organolépticas e estabilidade $(4,12,16,24,28)$.

Tendo em vista que no Brasil não existe legislação para a reutilização de óleos de frituras, alguns proprietários de lanchonetes e restaurantes usam o mesmo óleo por longos períodos.

Este trabalho teve como objetivo verificar a influência do tempo de reutilização do óleo de fritura, na aceitabilidade do produto frito, em nível de consumidor.

* Professor da Área de Alimentos do Centro Federal de Educação Tecnológica do Paraná- CEFET/PR-UNED-MEDIANEIRA.

** Professores do Departamento de Tecnologia Química da Universidade Federal do Paraná. 


\subsection{MATERIAL}

- óleo de soja refinado;

- Pastéis de queijo;

- Fritador de aço inox, tipo panelão, aquecido a fogo direto (gás), com capacidade para 6 litros de óleo.

\subsection{ANÁLISES FÍSICAS E QUÍMICAS}

- Viscosidade

A viscosidade foi determinada em viscosímetro de bolas (HOEPPLER), a temperatura de $18^{\circ} \mathrm{C}$, conforme manual do instrumento.

- Acidez e Índice de iodo

As análises foram realizadas segundo as normas analíticas do IAL(26). A acidez foi calculada em porcentagem de ácido oléico e o índice de iodo foi realizado pelo método de Hanus.

\subsection{ANÁLISE SENSORIAL}

Os pastéis foram elaborados na lanchonete da mesma maneira como são vendidos para o consumidor e fritos no mesmo óleo durante três dias consecutivos, em períodos ininterruptos de 12 horas de aquecimento diário e média de 400 frituras/dia. Durante esse período, o nível do óleo foi mantido pela reposição de um litro de óleo/dia. Para avaliação sensorial foi utilizada a escala hedônica de 9 pontos, sendo atribuído escore 9 (gostei muitissimo) e de 1 ao mínimo (desgostei muitissimo), permitindo-se entretanto aos julgadores o uso de valores fracionados. Cada provador recebeu um pastel de cada vez, durante os três dias de realização do teste, sendo que, a primeira avaliação ocorreu no dia em que o óleo havia sido trocado por volta das nove horas. A segunda após 18 horas de aquecimento, a terceira após trinta horas de aquecimento e a quarta e última avaliação, feita com pastéis fritos em óleo aquecido durante trinta e seis horas. o método da escala hedônica foi escolhido por ser o mais adequado para avaliar os os pastéis separadamente, já que estes foram fritos no mesmo óleo com diferentes intervalos de aquecimento e não podiam ser guardados para avaliação de uma só vez. os julgadores foram orientados para avaliarem principalmente 0 sabor e a aparência dos pastéis, em função da absorção de óleo em sua superfície.

Os valores obtidos foram submetidos aos testes estatisticos ANOVA e de TUKEY $(19,22)$. 
O Quadro 1 e a Figura 1 mostram algumas características físicas e químicas do óleo de soja usado no experimento.

QUADRO 1 - CARACTERÍSTICAS FÍSICAS E QUÍMICAS DO ÓLEO USADO NAS FRITURAS, EM DIFERENTES TEMPOS DE AQUECIMENTO

\begin{tabular}{|c|c|c|c|}
\hline $\begin{array}{c}\text { AMOSTRAS } \\
\text { Tempo de aque } \\
\text { cimento }(\mathrm{h})\end{array}$ & $\begin{array}{c}\text { ACIDEZ } \\
\%\end{array}$ & $\begin{array}{c}\text { VISCOSIDADE } \\
18^{\circ} \mathrm{C} \\
\text { ác.oléico }\end{array}$ & $\begin{array}{c}\text { INDICE } \\
\text { DE } \\
\text { IODO }\end{array}$ \\
\hline 1,5 & 0,09 & 11,00 & 127,29 \\
18,0 & 0,34 & 13,90 & 120,27 \\
30,0 & 0,54 & 18,04 & 114,52 \\
36,0 & 0,76 & 25,10 & 108,82 \\
\hline
\end{tabular}

A acidez e a viscosidade aumentaram com 0 tempo de aquecimento, enquanto o índice de iodo diminuiu, demonstrando a degradação que o óleo sofreu nesse período.

FIGURA 1 - CARACTERÍSTICAS FÍSICAS E QUÍMICAS DO ÓLEO USADO NA FRITURA DOS PASTÉIS

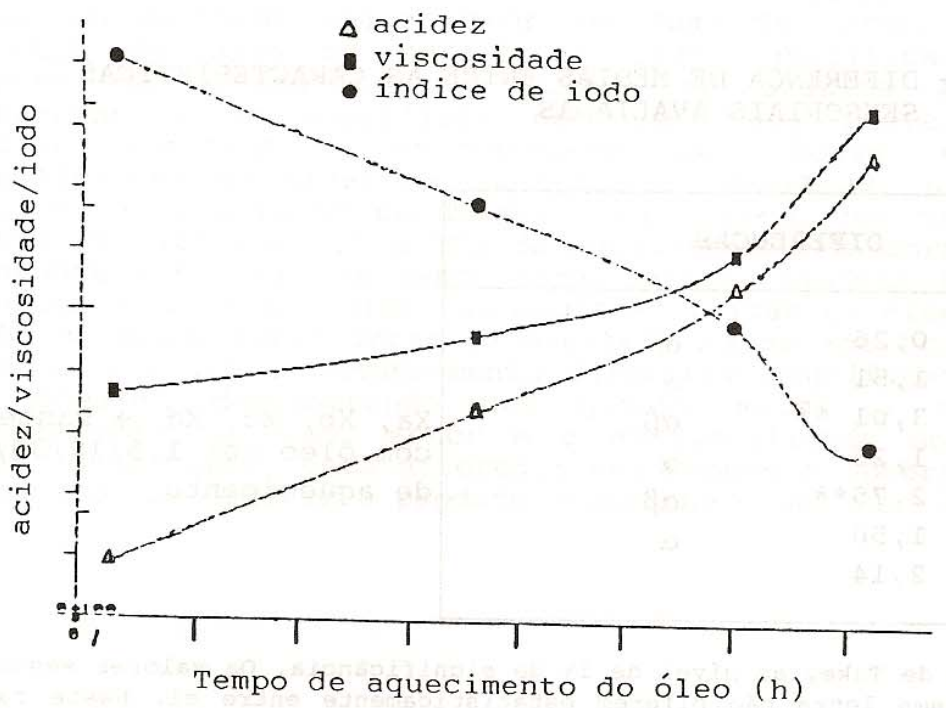

B.CEPPA, Curitiba, v. 13, n. 2, jul./dez.1995 
A Figura 2 e os Quadros 2 e 3 apresentam os resultados da análise sensorial.

FIGURA 2 - MÉDIA DAS NOTAS DOS JULGADORES ATRIBUIDAS AOS PASTÉIS

回 MÉDIAS DADAS PELOS JULGADORES

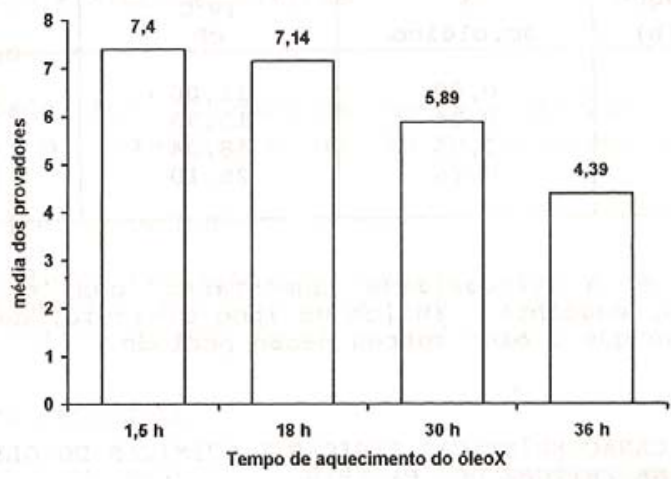

QUADRO 2 - DIFERENÇA DE MÉDIAS ENTRE AS CARACTERISTICAS SENSORIAIS AVALIADAS

\begin{tabular}{|c|c|c|c|}
\hline & DIFER & & \\
\hline $\begin{array}{c}X a-X b \\
X a-x c \\
X a-x d \\
X b-x c \\
X b-x d \\
X c-x d \\
1 \%\end{array}$ & $\begin{array}{l}0,26 \\
1,51 \\
3,01 * * \\
1,25 \\
2,75 * * \\
1,50 \\
2,14\end{array}$ & $\begin{array}{l}\alpha \\
\alpha \\
\alpha \beta \\
\alpha \\
\alpha \beta \\
\alpha\end{array}$ & $\begin{array}{l}\text { Xa, Xb, Xc, Xd } \rightarrow \text { Pastéis frit. } \\
\text { com óleo com } 1,5 / 18 / 30 / 36 \text { hola } \\
\text { de aquecimento }\end{array}$ \\
\hline
\end{tabular}

** Teste de Tukey ao nível de 18 de significăncia. Os valores seguidos da mesma letra não diferem estatisticamente entre si. Neste caso, as amostras consideradas diferentes apresentam diferença entre as médias superiores a 2,14 . 
QUADRO 3 - VALORES DOS QUADRADOS MÉDIOS E SIGNIFICÂNCIA DO TESTE F PARA AS CARACTERISTICAS SENSORIAIS DOS PASTÉIS DE QUEIJO FRITOS EM ÓLEO DE SOJA COM 1,5; $18 ; 30$ E 36 HORAS DE AQUECIMENTO

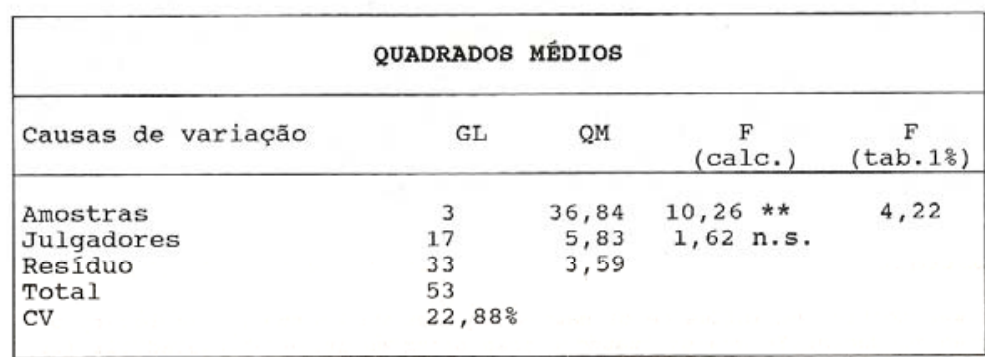

GL $=\quad$ Grau de liberdade

CV $=$ Coeficiente de variaçăo

** = Significância a nivel de 1 \%

n.s.= não significativo

Fcalc. $=$ F calculado

Ftab. = F tabelado

\begin{abstract}
As notas atribuidas às amostras pelos provadores foram decrescentes em função do tempo de aquecimento do óleo, demonstrando que os pastéis fritos em óleo aquecido por maís tempo apresentaram maior sabor residual de ranço, e maior absorção de óleo na superfície. Isto justifica-se pelo aumento da viscosidade do óleo, que resulta em maior encharcamento da superficie da massa dos pastéis. Pela analise estatistica constatou-se que houve diferenca analise que houve diferenç significativa ao nivel de $1 \%$ entre as amostras, $\circ$ que for confirmado pelo teste de Tukey. Verificou-se que os pastéis fritos em óleo com 1,5 e $18 \mathrm{~h}$ de aquecimento não apresentaram diferença entre si, da mesma forma que os pastéis fxitos em óleo com 18 e $30 \mathrm{~h}$. Porém, as amostras fritas em bleo com 1,5 e $30 \mathrm{~h}$ de aquecimento foram diferentes. Já as amostras fritas que quanto maior que quanto maior $\circ$ tempo de reutilização do óleo, menor é a aceitabilidade do produto frito. Entretanto, esta diferença nem sempre é observada pelo consumidor ao ingerir o produto acompanhado por refrigerante.
\end{abstract}


Os pastéis fritos em óleo aquecido até 36 horas consecutivas apresentaram aceitabilidade decrescente em função do tempo de aquecimento. As amostras de pastéis fritos em óleo com menor tempo de reutilização apresentaram menor sabor residual de ranço, melhor aparência e consequentemente maior aceitabilidade, ao contrário das amostras fritas em óleo com maior tempo de aquecimento. Estes resultados mostram que o óleo de fritura aquecido a fogo direto não deve ser reutilizado por mais de 30 horas de aquecimento constante, considerando o tipo de fritador e o sistema de aquecimento.

\section{Abstract}

It was tested the influence of heated soybean oil on the acceptability of fried products. It was evaluated cheese pastries fried on heated oil for 1,$5 ; 18 ; 30$ and 36 hours by a taste panel of eighteen people trained to use hedonic scale. The results showed that the heated oil couldn't be used for more than 30 hours of constant heat.

\section{REFÊRENCIAS BIBLIOGRÁFICAS}

1 ALMEIDA, L.A.S.B. et al. Batata pré-frita e hortaliças congeladas, economia e industrialização. Estudos econômicos-alimentos processados, Campinas, n. 18, p. 91, 1983.

2 ARAUJO, W.M., FUGMANN, A.J., CORREIA, R., FREITAS, R.J.S. Determinação dos índices de acidez e de peróxido em óleos vegetais submetidos ao calor no processamento de alimentos. Eng.Tecnologia, Curitiba, n. 4, p. 5-71, 1980 .

3 ARIMA, H.K. Gordura de fritura: a qualidade dos produtos na fritura. CTC-Tecnolcarnes, Campinas, v. 1, n. 6, 1985.

4 BARRERA-ARELLANO, D. Estabilidade em óleos e gorduras. óleos e grãos, S.Caetano do Sul, n. 13, p. 10-13, jul./ago., 1993.

5 BOBBIO, P. A., BOBBIO, F.O. Química do processamento de alimentos. São Paulo: Varela, 1992. 151 p.

6 EMÍlIo, S.C.G. Química e tecnologia de gorduras. Campinas : UNICAMP, 1988. 54 p.

7 FEDELI, E. The behaviour of olive oil during cooking and frying. In : FRYING of food. Chichester : Horwood, 1988. p. 52-81. 
8 FRANKEL, E. N. Recent advances in the chemistry of the rancidity of fats. In: BAYLEY, A. J. Recent advances in the chemistry of meat. [S.I] : The Royal Society of Chemistry, [198-]. p. 87-118.

9 FUGMANN, H.A., ARAUJO, W. M., FREITAS, R. J. S. Determinação do índice de peróxido e do índice de acidez de gorduras sólidas de origem animal, vegetal e vegetal hidrogenada submetidas ao calor no processamento de alimentos. Eng.Tecnologia, Curitiba, n. 5, p. 43-58, 1982 .

10 GILLATT, P. N. , ROGERS, E. K. , ROSSELL, J. B. Development and evaluation of a novel sensor for the in situ assessment of frying oil quality. Food Control, July, 1990.

11 GIOIELLI, L.A., PITOMBO, R.N. de M., SAKATE, M.S. Efeito da atividade de água na formação de compostos voláteis durante a oxidação do óleo de amendoim bruto. Ciência e Tecnologia de Alimentos. Campinas, v. 12, n. 1, p. 1-98, jan./jun. 1992 .

12 GOODMAN, A. H., BLOCK, Z. Problems encontered in the commercial utilization of frying fats. JAOCS, v. 29, p. 616-619, 1952 .

13 ISKANDER, M. H. Effect of heating on some chemical characteristics, fatty acid composition and stability of corn and palm oils. La Rivista Italiana Delle Sostanze Grasse, Minia Egypt, v. 68, p. 421-432, ago. 1991.

14 KEPPLER, J.G. Twenty-five years of flavor research in a food industry. JAOCS, Champaign, v. 54, n. 10, p. 474-7, 1977.

15 LILLARD, D. A. Effect of processing on chemical and nutritional changes in food lipids. J.Food Protec, Ames, v. 46, n. 1, p. 61-7, 1983.

16 MAGOFFIN, J. E., BENTZ, R. W. The use of antioxidants in potato chipping. JAOCS, v. 26, p. 687-690, 1958.

17 MAYNARD, A., JOSLYN, M. S., Food Processing operations. London : Avi, 1964. V. 3

18 MCDOWELL, D. A., FITZ, F. Health implications of the application of polynsatured fatty acids in deep-fat frying In: FULLER, D. B. J., DARRY, R. T. Savoury coatings. London: Elsevier, 1987. $130 \mathrm{p}$.

19 MORAES, M. A. C. Métodos para avaliação sensorial dos alimentos. 7.ed. Campinas, 1990. $93 \mathrm{p}$. 
20 NAVEIRA, R. M. L. P., OLIVEIRA, C. P., GLORIA, M. B. A. Quality assessment of frying oils used for "palha" type fried potatoes. Belo Horizonte : Food Department, Faculty of Pharmacy, Federal University of Minas Gerais, [198-]. p. 209-15.

21 NAWAR, W. W. Lipids. In: FENNEMA, O. R. Food Chemistry. 2.ed. New York : Marcel Dekker, 1985. p. 139-244.

22 O'MAHONY, M. Sensory evaluation of food : statistical methods and procedures. New York : Marcel Dekker, 1986. $487 \mathrm{p}$.

23 PAQUETTE, G., KUPRANYCZ, D. B., VAN DE VOORT, F. R. The mechanisms of lipid autoxidation. I. Primary oxidation products. Can. Inst. Food Sci. Technol., v. 18, n. 2, p. $112-8,1985$.

24 POLING, C. E., WARNER, W. D., MONE, P. E., RICE, E. E. The influence of temperature, heating time and aeration upon the nutritive value of fats. JAOCS, v. 39, p. 315320,1962 .

25 PREVOT, A., DESBORDS, S. MORIN, O., MORDRET, F. Volatiles and sensory effect from frying oils. In : FRYING of food. Chichester : Horwood, 1988. p. 155-165

26 INSTITUTO ADOLFO LUTZ. Normas Analíticas do Instituto Adolfo Lutz (IAL). São Paulo, 1976. 371 p.

27 SHIROSE, I., FERREIRA, V.L.P., AGUIRRE, J.M. Estimativa da vida de prateleira de soja frita com base no delineamento parcialmente escalonado. Col.ITAL, v.9, 1978 .

28 STERN, S., ROTH, H. Properties of frying fat related to fat absortion in doughnut frying. Cereal Sci. Today, v. 4 , p. 176-179, 1959 .

29 SULTHANA, S. N., SEN, D. P. Studies on deep fryingchanges during heating of oil. Journal of Food Science and Technology, v. 16, Sep./Oct. 1979.

30 TANGO, J. S., ANDRADE, E. F., SHIROSE, I. Alterações nos óleos de castanha do pará, amendoim, algodão e arroz, durante o processo de fritura de batatinha. Col.ITAL, v. 8, p. $75-94,1977$. 